\title{
Wavefield Characterisation of MHz XFEL Pulses
}

\author{
Trey Wilson Guest ${ }^{1,2}$, Brian Abbey ${ }^{1}$, Adrian Mancuso $^{1,2}$, David Paganin ${ }^{3}$, Richard Bean ${ }^{2}$, Grant van Riessen ${ }^{1}$ \\ ${ }^{1}$ La Trobe Institute for Molecular Sciences, La Trobe University, Victoria 3086, Australia.; \\ ${ }^{2}$ European XFEL GmbH, Albert-Einstein-Ring 19, 22761 Hamburg, Germany; \\ ${ }^{3}$ School of Physics, Monash University, Victoria 3800, Australia \\ twguest@students.latrobe.edu.au
}

Interpretation and analysis of XFEL data can depend critically on a fundamental understanding of the characteristics of the XFEL pulses. To exploit the unique repetition rate of the EuXFEL requires understanding of both the inter- and intra-train fluctuations in pulse fluence, spatial energy distribution, coherence and wavefront, and beam pointing, which are frequently implicated in the loss of information in XFEL single particle imaging (SPI) and other classes of coherent diffraction experiment [1], [2] . Failure to account for fluctuations of the electron bunch phase-space and/or trajectory within a pulse train can result in deviations of the recorded wavefront, intensity statistics and intensity integrals from theoretical behaviour.

Preliminary X-ray optical data collected at the SPB-SFX instrument of the European XFEL demonstrates a sensitivity of inter- and intratrain variations in beam pointing to different beam delivery parameters [Fig. 1.]. We present this data alongside a model of the SASE1 photon beam. A partially coherent wave optical simulation of the beam propagated from the undulator exit to the SPB-SFX instrument hutch is compared to experimental data collected in the same plane, and discuss the design of wavefront measurement methods that can be made for comparison with theory. Moving forward, we outline a novel method for investigating the relationships between the statistical behaviour of the XFEL source (including inter- and intra-train jitters) and the optical wavefrontproperties observed at the instrument to extend these observations.

[1] Z. Sun, J. Fan, H. Li, en H. Jiang, “Current Status of Single Particle Imaging with X-ray Lasers”, Appl. Sci., vol 8, no 1, bl 132, Jan 2018, doi: 10.3390/app8010132.

[2] D. Oberthür, "Biological single-particle imaging using XFELs - towards the next resolution revolution", IUCrJ, vol 5, no 6, bll 663-666, Nov 2018, doi: 10.1107/S2052252518015129.

Keywords: Single Particle Imaging, X-ray Free Electron Lasers, Wavefront Characterisation, Phase-Retrieval 